

\section{SALUD PÚBLICA EN MÉXICO IMPLICACIONES PARA LA ENSEÑANZA DE LA BIOLOGÍA Y LAS CIENCIAS DE LA SALUD*}

\section{Public health in Mexico Implications for Biology teaching and Health Sciences}

Jesús Carlos Ruvalcaba Ledezmaํ / Sandra Yazmín Cortés Ascencio² / José Margarito Jiménez Mora

Fecha de recepción: 16 de febrero de 2013 Fecha de aprobación: 22 de junio de 2013

\section{Resumen}

La salud pública se define como la ciencia y arte de impedir las enfermedades, prolongar la vida y fomentar la salud y eficiencia mediante el esfuerzo organizado de la comunidad. La salud pública surge en nuestro país como una necesidad ante las condiciones sociales y culturales. La introducción de la vacuna antivariolosa, el consejo general de salubridad e instituciones de salud enmarcan el inicio de la salud pública en nuestro país, las acciones implementadas en salud pública y el crecimiento demográfico, entre otros determinantes socio-ambientales de la salud, han provocado cambios en el estilo de vida; la tasa de mortalidad ha disminuido y la esperanza de vida al nacer se incrementó sustancialmente, por lo que se requiere enfrentar los retos de la transición de las enfermedades infecciosas y parasitarias a las enfermedades crónicas, bajo la comprensión de la perspectiva educacióncultura socio-ambiental y mediante recursos humanos altamente calificados en salud pública. Esto requiere que en el proceso enseñanza-aprendizaje de la biología sea necesaria la aplicación del enfoque constructivista, desde diversos modelos, entre ellos el de la modificabilidad cognitiva en el contexto-realidad del alumno, para generar científicos con mayor capacidad para la resolución de problemas de investigación biológica y, por consecuencia, mejores estrategias de impacto en la salud pública de México.

Palabras clave: salud pública, educación-cultura, socio-ambiental, constructivismo-modificabilidad cognitiva.

\section{Abstract}

The public health is defined as the science and art to prevent the diseases, prolong the life and promote the health and efficiency by means of the organized effort of the community. The public health arises in our country as a necessity in the face of the social and cultural conditions. The introduction of non valorous vaccine, the general council of healthiness and institutions of health frame the beginning of the public health in our country, the actions implemented in public health and the demographic growth, among others socio-environmental factors have provoked changes in lifestyle, mortality rate has decreased and the life expectancy has increased substantially, that's why it is important to face the challenges of transition of infectious and parasitic diseases to the chronic diseases, with the understanding of the education, socio-environmental culture and by means of highly qualified human resources in public health. This requires that in the teaching-learning process of biology the application of the constructivist approach is required, from different models, including cognitive modifiability in the context and reality of the student to generate scientists with more capacity to solve biological research problems and consequently, better strategies for public health impact of Mexico.

Keywords: Public Health, Education-Culture, Socio-Environmental, Cognitive Adjustability Constructivism.

* Artículo derivado de una línea de investigación.

1 Doctor en Ciencias de la Salud Pública, Profesor Investigador de Tiempo Completo Área Académica de Medicina, Profesor de Introducción a la Salud Pública y Epidemiología. ICSa-UAEH Instituto de Ciencias de la Salud--Universidad Autónoma del Estado de Hidalgo, Pachuca Hidalgo, México. Correo electrónico: dcspjcarlos@hotmail.com

2 Maestría en Ciencias de la Salud, Profesor en la Universidad La Salle Pachuca Hidalgo, México, Doctorante en Ciencias Ambientales ICBI-UAEH Instituto de Ciencias Básicas e Ingeniería--Universidad Autónoma del Estado de Hidalgo, Pachuca México. Correo electrónico: ss1ale@hotmail.com

3 Maestría en Investigación Educativa, Profesor investigador posgrados en Investigación Educativa U de G Universidad de Guadalajara, México. Correo electrónico: pepe_dw@hotmail.com 


\section{Introducción. La salud pública, un concepto en evolución}

Dos grandes problemas de la educación corresponden a la baja calidad del proceso enseñanza-aprendizaje y la desigualdad educativa; el primero debe involucrar al alumno y al maestro en la construcción del conocimiento, el desarrollo de habilidades-competencias, capacidades, destrezas, así como actitudes y valores, que garantice la participación activa en su proceso social, de su comunidad y desarrollo de su país. Por lo mismo, se requiere profesionalización del docente, actualización innovadora para la construcción del conocimiento. Partiendo de esta implicación de la educación como proceso transformador del pensamiento (González, 1999), iniciamos este bioensayo a partir del concepto fundamental de la salud pública en su historia y la incorporación de la enseñanza de la biología-la salud pública y, finalmente, la propuesta educativa desde la perspectiva constructivista con énfasis en la teoría de la Modificabilidad Cognitiva (Feuerstein, 1994 y Velarde, 2008).

La salud pública puede definirse como la ciencia y arte de impedir las enfermedades, prolongar la vida y fomentar la salud y eficiencia mediante el esfuerzo organizado de la comunidad (López, 1993). Realizando un análisis de la definición, encontramos que implica un componente preventivo al referirse como la ciencia y arte de impedir las enfermedades, abarcando acciones que enmarcan su detección oportuna. Por otra parte, el concepto también comprende un componente de promoción, cuya intencionalidad es fomentar la salud y eficiencia mediante el esfuerzo organizado de la comunidad para: el saneamiento del medio donde se incluye; aprovisionamiento de agua, disposición adecuada de excretas, saneamiento de vivienda, saneamiento de alimentos y control de fauna transmisora o nociva, asimismo tomar en cuenta el control de enfermedades transmisibles, la educación de los individuos en higiene personal, la organización de los servicios médicos y de enfermería para el diagnóstico temprano y el tratamiento preventivo de la enfermedad y el desarrollo de un mecanismo social que asegure a cada uno un nivel de vida adecuado para la conservación de la salud. En el entendido de la preposición para como destino o propósito, y en este punto se identifican las funciones de la salud pública, la accesibilidad y la calidad de los servicios de salud, y, finalmente, un componente de autogestión, entendido como la participación de la comunidad en su propio desarrollo, en la mejora de sus condiciones socio-ambientales y de salud pública.

Lo anterior se refiere a la definición de salud pública propuesta por Winsolow, 1920 donde ofrece por primera vez una perspectiva de la salud pública moderna. La conceptualiza en su contexto sanitarista y como un movimiento social para lograr no solo mejores niveles de salud, sino también mayor equidad en salud. Propone una salud pública amplia y diversa, que incluye el diagnóstico temprano y la prevención, así como la organización de los servicios médicos dirigidos a la persona. Winslow señala que la salud pública se reconocía como una ciencia emergente, óptima y cambiante, que abarcaba tanto aspectos del control de enfermedades infecciosas y la prevención de enfermedades crónicas y mentales como el control de enfermedades asociadas a la pobreza. (Winslow, 1923).

En la actualidad, el objetivo de la salud pública (utilizando el término pública como aquello que interesa a todos y se enfoca al bien común de la sociedad) es mejorar la salud y el bienestar de la población. Las acciones de salud pública inciden sobre los determinantes sociales que condicionan el nivel de salud; la prevención de epidemias y el control de enfermedades; la protección contra riesgos ambientales, la prevención de daños a la salud, la promoción y fomentos de conductas saludables, la respuesta organizada y oportuna a los desastres, y la garantía de calidad y accesibilidad a los servicios públicos de salud. Adicionalmente, en muchos países se les asignan diversas responsabilidades para mitigar el efecto asociado a nuevas tecnologías de salud, a la globalización y la migración; y la prevención y preparación ante posibles actos de bioterrorismo (Lazcano et al., 2013). El objetivo incide en los problemas de salud de las comunidades desde un nivel poblacional bajo un enfoque transdisciplinario e integrador, la esencia de la salud pública moderna desde una perspectiva poblacional que inspira dos vertientes, una como punto de encuentro de diversas disciplinas y áreas del conocimiento y otra como ámbito de acción para maximizar la salud de las colectividades (Frenk, 2003).

El auge de la salud pública ha tenido un impacto importante en el mejoramiento de las condiciones de vida a nivel mundial, así como en la denominada transición epidemiológica. Las acciones emprendidas en salud pública en el mundo han tenido un efecto significativo en el desplazamiento de las tasas de morbi-mortalidad, aumentando la esperanza de vida al nacer. Sin embargo, la atención a esta situación pone de manifiesto cómo también han surgido enfermedades con una nueva presentación en la sintomatología ante los estudiosos de la medicina. También se observa el desarrollo de las enfermedades crónicas como una forma del desplazamiento de las enfermedades infecciosas y las parasitarias, entre otras. Esto puntualiza que, aunque se continúa prestando atención a las enfermedades infecciosas, las de principal impacto ahora corresponden a las enfermedades crónicas, por lo que es necesario analizar la postura de la salud pública de manera histórica (López, 1993 y Frenk, 2007). además de poner énfasis en la corresponsabilidad que en salud pública se requiere hoy en día para lograr abatir tantos problemas que requieren de la colaboración del gobierno, así como de la participación de los miembros de las comunidades (Cortés, 2011a). 


\section{Génesis y desarrollo de la salud pública en México. Una síntesis}

La medicina primitiva era empírica y mágica, ya los aztecas conocían algunas enfermedades de tipo infeccioso como la viruela y el sarampión, el tifo y el paludismo. Con la llegada de los españoles, llegan otras enfermedades vinculadas con mecanismos de transmisión sexual como la sífilis, para la cual se utilizaron tratamientos a base de temazcalli y sales de mercurio, e incluso la aplicación de plantas medicinales como el achiote para la lepra (López, 1993). Uno de los acontecimientos que impactan ya en 1804 en ciencia y tecnología en México fue la vacuna (antivariolosa) contra la viruela, la misma que marca la pauta del inicio de la aplicación de medidas o acciones de salud pública en la población. Luego de dos siglos, otra novedad más se aprecia en la transición epidemiológica, es decir, en el cambio y frecuencia de las enfermedades infecciosas y parasitarias a la proliferación de las enfermedades crónicas como principal causa de muerte (López, 1993).

Entre los acontecimientos más importantes del México poscolonial, resalta en enero de 1841 la creación del Consejo Superior de Salubridad del Departamento de México, tomando el lugar del protomedicato (López, 1993) de la facultad de medicina de 1831. Entre las funciones de este consejo se encontraba la de intervenir en asuntos de higiene pública y certificar títulos para el ejercicio de la medicina, así como de las labores de parteras y dentistas, la supervisión de venta de medicamentos y el funcionamiento de las boticas. En 1891 se dicta el primer código sanitario. A principios del siglo $X X$, en 1902 , se lucha por la erradicación de la fiebre amarilla y del paludismo, y para 1907 se inician las campañas contra la sífilis. Durante la siguiente década, se decretan las bases de seguridad social y se publica por Rafael Lucio Nájera Breves la obra Consideraciones sobre las condiciones higiénicas de las maternidades, además de un primer estudio sobre la lepra y la fiebre amarilla. El doctor Eduardo Liceaga, en 1920, reinstala el Instituto Bacteriológico Nacional en el Distrito Federal, dedicado a tareas de salud pública, como la lucha para erradicar la fiebre amarilla, la vacuna contra la hidrofobia y una política del control de epidemias (López, 1993).

Fue también en los primeros años de este siglo que el doctor José María Rodríguez fue nombrado presidente del Consejo Superior de Salubridad, y en octubre de 1917 funda el Cuerpo Médico Militar, con el cual abrió la posibilidad de vacunar en forma masiva contra la viruela y, posteriormente, a su erradicación. En 1922, se inaugura la escuela de salubridad y se reestructura en 1925, contando con su código sanitario para 1926; para 1929, da inicio la Asociación Nacional de Protección a la Infancia y en 1931, la Ley Federal del Trabajo, en la cual se consideran accidentes y enfermedades profesionales; en 1934, se da inicio a la campaña antituberculosa, se crea el Instituto Politécnico Nacional con la Escuela de Medicina y para 1936 se establece el servicio social a estudiantes de medicina, mientras que en 1939 se inaugura la Secretaría de Salubridad y Asistencia (SSA), de la cual procede la actual Secretaría de Salud.

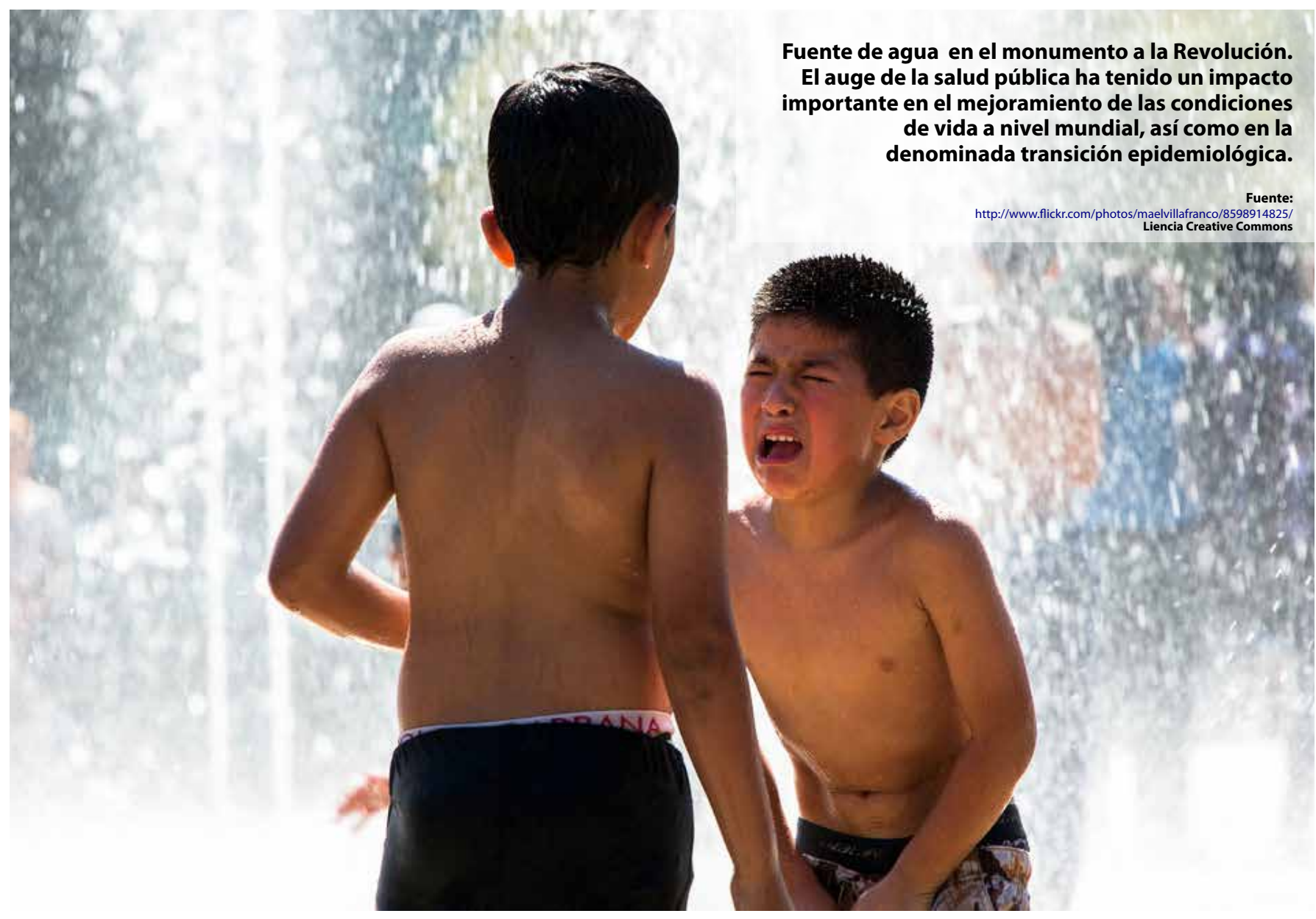


A mediados del siglo mencionado, se creó el Hospital de Enfermedades de la Nutrición, en 1944, y para 1951 se había erradicado la viruela. Dos años después, se había creado el Programa de Bienestar Social Rural y en 1955, el nuevo código sanitario. Por otro lado, uno de los eventos más importantes es que la Escuela de Salubridad se transformó en la Escuela de Salud Pública (López, 1993 y Fajardo, 2002).

Durante el periodo 1959-1964, la Secretaría de Salud incorpora a su estructura áreas hospitalarias y centros de salud, se aplican para su erradicación vacunas contra poliomielitis, tifo y progresan campañas para la erradicación de la malaria, entre otras; para 1956, se aplican campañas a nivel nacional en nuestro país para combatir la poliomielitis, difteria, tosferina, tétanos, tuberculosis, fiebre reumática, mal del pinto, oncocercosis, lepra y otras enfermedades de la piel, bocio, accidentes y acciones contra el mosquito Aedes aegypti.

En 1959, a su vez, inicia el funcionamiento del Instituto de Seguridad y Servicio Social para los Trabajadores del Estado (ISSSTE) y el Instituto Mexicano del Seguro Social (IMSS). En el periodo que comprende los años 1965 a 1970, la salud pública se ejerce con la filosofía de la promoción de la salud, con énfasis en la prevención, curación y rehabilitación de las enfermedades. En 1973, el IMSS amplía su cobertura e instituye el programa solidaridad social y la Secretaría de Salud amplía su red hospitalaria, se dio a conocer el Plan Nacional de Salud y en 1979 se instituye la cartilla nacional de vacunación (López, 1993).

Durante la década de los ochenta, se emiten disposiciones sanitarias como el certificado prenupcial, que incluye la realización de pruebas de tipo sanguíneo y VDRL, entre otras. En 1980, se incrementaron los programas de salud rural y de vacunación contra la tuberculosis y para 1983 se elevó a categoría constitucional el derecho a la protección de la salud para todos, vinculándolo con las garantías sociales.

En los años noventa, se creó el programa IMSS-Solidaridad y en 1991 se registran los últimos casos de difteria y de poliomielitis. En 1997, se diseñó el Programa de Educación y Salud (Progresa), destinado a llevar atención social a las zonas marginadas, preponderantemente. En nuestro país se adopta, acorde con la Organización Mundial de la Salud (OMS), la frase "Salud para todos" en el 2000 (López, 1993) y Fajardo, 2002).

A inicios del nuevo siglo, en el 2000, el programa Oportunidades remplaza al programa Progresa, entonces se inician los comentarios y predicciones respecto a que estamos a punto de enfrentar una de las epidemias más serias en la humanidad, la obesidad y problemas metabólicos, incremento en diabetes mellitus tipo 2, problemas cardiovasculares, violencia y accidentes, situaciones que se agravaron hacia 2010, cuando se conoce que México se encuentra en los primeros lugares a nivel mundial en problemas de obesidad general y con alarma particular en obesidad infantil, en niños que de seguro traerán ya una carga extra de riesgo para algún problema metabólico, incluyendo diabetes o incluso algún tipo de cáncer.
Para 2002, la Organización Panamericana de la Salud (OPS) cumple cien años de existencia y resulta inevitable pensar la desigualdad imperante entre desarrollo, educación y salud pública en las distintas regiones, por lo que existe la necesidad de concebir una relación recíproca entre los niveles de salud y el grado de desarrollo económico de una sociedad. La salud depende de la accesibilidad a los servicios, a satisfactores básicos que definen la calidad de vida, al tiempo y al desarrollo económico, al esfuerzo colectivo, en fin, es un punto donde se vinculan la política social con las metas de bienestar, y la política económica, con su interés por la producción (Castillo, 1998 y Frenk, 2003).

\section{Realidad u oportunidad para la salud pública}

El panorama actual hace evidente que en algunos estados de la República Mexicana, entre ellos Oaxaca, Chiapas, Guerrero y otros del sur del país, la transición epidemiológica no es muy clara, no permite ver la separación entre el tipo de enfermedades que en los años cincuenta representaron la principal causa de muerte, tales como la diarrea, las enfermedades contagiosas y parasitarias de las enfermedades crónicas, ya que existen comunidades cuyo nivel social y económico es muy bajo, debido en buena medida a su dispersión geográfica. Ambos factores son determinantes para que estas regiones sigan sufriendo de enfermedades que en el resto de la República aparentemente han dejado de ser consideradas como las principales causas de muerte, tal es el caso de la amibiasis, los casos de parasitosis por Ascaris lumbricoides, Onchocerca volvulus, Taeniasis y otras transmitidas por vector, las cuales continúan causando estragos en la población humana, como es el caso del dengue y de la enfermedad de Chagas.

Resulta preocupante, además, en lo que se refiere a la relación entre educación y salud, tener estándares en educación por debajo de lo que la población requiere. En estados como Oaxaca, donde los niveles educativos son los más pobres del país, entre los principales problemas de salud pública se encuentran la mortalidad materna, la malnutrición, las enfermedades crónicas como el cáncer, las enfermedades de transmisión sexual y aquellas transmitidas por vector. Así, la situación socioeconómica y las condiciones de educación interfieren en el desarrollo de sus comunidades, lo cual, como se ha mencionado, junto a su dispersión geográfica, acarrea dificultades adicionales de acción y ejecución de la salud pública en el acceso a los servicios de salud.

En este sentido, existe una vinculación directa entre educación, cultura, entorno y salud pública. Algunas de las enfermedades que prevalecen en regiones como la señalada en el párrafo anterior, como se ha mostrado, están asociadas a la situación socioeconómica, ambiental y genética prevaleciente en las comunidades. Otros padecimientos como la tuberculosis, gastroenteritis diarreica infecciosa, dificultades respiratorias, y una amplia gama de enfermedades crónicas, son solo un ejemplo de que en zonas donde las condiciones sociales son más pobres, se reproducen males que podrían mitigarse o erradicarse mediante esfuerzos colectivos de transformación, en los cuales tiene un lugar fundamental la educación. Es urgente entonces que la educación y la cultu- 
ra adquieran nuevas dimensiones de desarrollo, de acuerdo con el contexto socio-ambiental en el que se impulsa la salud pública. Algunas alternativas sobre este punto son discutidas enseguida.

\section{La educación como factor de cambio}

Un aspecto fundamental para el desarrollo en salud pública, como se ha planteado anteriormente, es la educación. Sin embargo, el modelo educativo imperante en nuestro país parece no tener en cuenta la necesidad de generar -de manera eficaz y permanente- en las aulas y las escuelas una reflexión crítica sobre la realidad. En contraste, aunque se proponen directrices para la educación como las derivadas de la concepción constructivista y el aprendizaje significativo (Díaz Barriga y Hernández, 2003), o la educación basada en competencias (Bogoya et al., 2004), la realidad en la aulas es que se sigue privilegiando un modelo tradicional donde el conocimiento se intenta reproducir y, en el mejor de los casos, recrear los procesos que le dan lugar, pero tal conocimiento está lejos de adquirir una condición fundamental, señalada por teóricos como Zabala (2006): que el conocimiento sirva para comprender el mundo, o, como ha señalado de manera más reciente Monereo (2010), que el fin último de la enseñanza, por encima de la formación de competencias, sea la resolución de problemas de la realidad.

Partiendo de esta problemática, en el caso concreto de la biología, si en su enseñanza se ejecuta un modelo repetitivo, memorístico, entonces no puede apreciarse de manera cabal el desarrollo y los descubrimientos que por parte de los biólogos, los químicos y médicos se han dado y, sobre todo, la trascendencia de sus aportaciones. Constantemente, escuchamos noticias sobre el calentamiento global, el cambio climático y temas afines, pero desde el punto de vista educativo no hemos sido capaces de incidir en la creación de una conciencia individual y colectiva que derive en acciones de transformación y mejora sobre un mundo del cual conocemos los problemas biológico-ambientales, pero no somos proactivos para determinar las soluciones.

Como han hecho notar diversas críticas (Ruvalcaba, 2002 y Cortés, 2011a), el modelo educativo tradicional no contribuye a desarrollar en el educando habilidades para pensar, para cuestionar no solo la realidad, sino a sí mismo, y para construir el conocimiento, competencias todas ellas que resultan fundamentales en la formación y en la vida de todos los seres humanos. De ahí la importancia de integrar un enfoque de la educación donde el conocimiento sea un medio, y no un fin, que sirva para comprender y transformar el mundo, y de donde se deriven, entre sus mejores efectos, conductas saludables (Cortés, 2011b). Siguiendo a Bonilla y Marín (2011), el hombre es el mayor agente de transformación y de modelación del mundo natural, por lo que se requiere analizar la composición de sus actitudes y, en general, de su compromiso con el medio ambiente. La relación entre individuo, cultura, naturaleza y sociedad tiene que ser uno de los contenidos básicos de cualquier propuesta educativa dirigida a formar personas responsables con su medio ambiente.
La orientación de la educación sobre bases como las anteriores permitiría cambios en la actitud, provocando una conciencia crítica en las personas. Por ello, es importante que se modifique tanto el modelo educativo como las oportunidades de acceso a educación de calidad, es necesario que se implemente modelos pedagógicos que generen o provoquen en las personas o educandos la habilidad para pensar, para cuestionar su realidad, y con ello, obviamente, se tendrán mejoras en salud pública, que por tanto llevarían a las personas más vulnerables a experimentar nuevas formas de resolver sus problemas.

La educación y la salud pública, desde una perspectiva biológica y social, se encuentran vinculadas directamente, en el marco entorno-cultura. Por tanto, la base de una transformación en las condiciones de salud pública en una comunidad se encuentra tanto en principios biológicos como el respeto por el medio ambiente y el cuidado de la salud individual como en principios sociales; por ejemplo, el de la participación en tareas en las que los esfuerzos compartidos generan beneficios comunes. Crear conciencia ecológica es una de las finalidades básicas que se desprenden de adoptar esta perspectiva (Ruvalcaba, 2010).

En este sentido, para quienes trabajamos desde la trinchera de las aulas, es conveniente propiciar que el alumno adquiera la capacidad de cuestionarse y de cuestionar su realidad. Solo de esta manera pensará, le surgirán dudas que lo lleven a plantear problemas de investigación y a la búsqueda de soluciones a la problemática ecológica de su entorno.

\section{La necesidad de un modelo de praxis del conocimiento}

Las reflexiones anteriores conducen a admitir que la salud pública se ha vuelto un área especializada de conocimiento e investigación. Por tanto, se espera que los estudiantes, en primer lugar, aprecien este carácter. En lo que se refiere a la biología, esta ha ocupado un lugar determinante en la historia de la salud pública, pues es el complemento del conocimiento médico en la atención a los problemas de salud que se han dado durante las épocas que se han recorrido.

De aquí se desprende la necesidad de un modelo para la enseñanza que privilegie la relación entre la teoría y la práctica. Paulo Freire, uno de los pedagogos clásicos, refiere en sus diversas obras (Freire, 1981, 1986, 1991) cómo la teoría sin la práctica se vuelve un verbalismo, mientras que la práctica sin teoría se vuelve un activismo irreflexivo. Como forma de evitar estos extremos en la enseñanza, propone el concepto praxis, cuyo contenido fundamental es justamente la realimentación entre estos dos productos de la experiencia humana. Se trata, en palabras del gran pensador, de conseguir la acción mediada por la reflexión. En el caso de la biología, y en general de las ciencias de la salud, si su aprendizaje se desprende de las partes de la realidad que les dan origen, se vuelve un conocimiento inútil. Por el contrario, se tiene que favorecer durante la enseñanza la posibilidad de que este conocimiento transite de su fuente (los fenómenos que tienen lugar en los seres vivos) a sus aplicaciones y áreas de oportunidad, como se muestra en el esquema siguiente: 
Figura 1. Proceso enseñanza de la biología y su aplicación en investigación, de la fuente a la praxis.

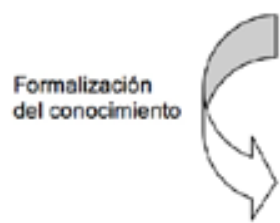

OB.JETOS DE LA REALIDAD

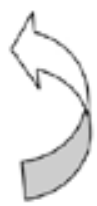

aplicación e investigación

DISCIPLINA DE LA BIOLOGIA

Fuente: Construcción colegiada por Ruvalcaba Ledezma, Cortés Ascencio y Jiménez Mora, 2012

Un modelo de trabajo que facilite esa vinculación, como el que hemos venido poniendo en práctica en el nivel medio superior y superior, tiene como base el principio anterior, según el cual el conocimiento de la biología procede de los seres vivos y su medio y, por tanto, se resignifica ante situaciones reales y adquiere sentido como objeto de enseñanza en la medida en que resulta útil al estudiante para comprender el mundo y sus fenómenos. Asimismo, retoma la idea compartida por Perrenoud (2002), Morin (2007) y Monereo (2010) de que son justamente los problemas que enfrentan estudiantes y profesores dentro y fuera del aula, $y$ no las competencias per se, lo que tiene que estar a la base de las diversas propuestas educativas. En este sentido, el modelo que proponemos, en el que se incluye de manera fundamental el análisis de problemas actuales en la cuestión de salud individual y del deterioro ambiental y, de manera paralela, la revisión de los diversos esfuerzos históricos en materia de salud pública, tiene tres grandes momentos:

\section{Comprensión científica de la problemática}

De acuerdo con Sigüenza y Sáez (1990), en el caso de la biología, un problema en el contexto del aula puede definirse como una situación cuya solución requiere que el sujeto analice unos hechos y desarrolle razonadamente una estrategia que le permita obtener unos datos (numéricos o no), procesarlos (relacionarlos entre sí y con los hechos), interpretarlos y llegar a una conclusión (respuesta). Este análisis y razonamiento debe basarse en la comprensión del tema o del campo al que pertenece la situación. Así, como parte de este primer momento del modelo de trabajo que proponemos, se define una situación a analizar mediante el conocimiento de la biología, que puede ser de carácter personal (por ejemplo, la contracción de una enfermedad de transmisión sexual), o ambiental (los efectos de un canal de aguas negras a cielo abierto sobre la población aledaña en una zona urbana). Se trata de pasar de los modos de explicación de sentido común a lo que Pozo (1989) denomina , es decir, a la incorporación de ideas y conceptos propios de la disciplina relacionados con los elementos que conforman tales situaciones problemáticas. En el primer caso, el de carácter individual, se tendría que aludir a una serie de conocimientos biológicos y de salud, como los síntomas de determinadas infecciones adquiridas por contacto sexual, mientras que en el segundo caso, referido a una problemática colectiva, los estudiantes tendrían que acceder a conocimientos relacionados con los posibles microorganismos que sobreviven en el aire como resultado del paso de aguas negras cerca de su comunidad.

\section{Acopio de información empírica}

Recordando que disciplinas como la biología, la medicina y la salud pública tienen su fuente de datos principal en la propia realidad, es la experiencia de investigar, de hacer trabajo de campo, la que permite obtener a los científicos que han contribuido a la evolución y al reconocimiento social de estas disciplinas, la que de alguna forma es necesario re-crear en los estudiantes. Una vez revisados en el aula los conceptos y esquemas en torno a problemáticas como las ejemplificadas en el momento anterior, se vuelve necesario que busquen datos en su medio para corroborar la presencia de aquello que han seguido de manera teórica. En el primer caso que se cita, se tendría que utilizar un instrumento como el cuestionario para determinar, en la población estudiantil, por ejemplo, el número de personas que tienen actividad sexual no protegida, y si eventualmente han experimentado determinados síntomas producto de ciertas infecciones. En el segundo caso, se tendría que ir a la zona cercana al canal de aguas negras y tomar muestras de aerosoles, para luego analizar en el laboratorio la presencia de enterobacterias y otros microorganismos patógenos. Es esta recreación de la experiencia científica la que permite sacar a la biología de un carácter de conocimiento inerte hacia uno de conocimiento vivo, en permanente cambio, incremento y evolución. Asimismo, da lugar a la posibilidad de que los alumnos reconozcan las características de los modelos o teorías explicativas utilizadas por la ciencia para los fenómenos sobre los cuales recogen datos. Estas características son sintetizadas en el trabajo de Felipe, Gallarreta y Merino (2005) en las siguientes afirmaciones:

- Son construcciones de la mente humana, por tanto, de naturaleza temporal, pueden evolucionar.

- Son representaciones de ideas o conceptos que se tienen sobre algún aspecto de la realidad.

- Son uno de los principales productos de la ciencia y cumplen un importante papel en la construcción del conocimiento y la comprensión de los fenómenos naturales.

- Proveen representaciones de ideas y conceptos presentados en una teoría.

- Ayudan a los científicos a predecir, describir y explicar fenómenos naturales, objetos y estructuras.

\section{Reinserción del conocimiento}

Este momento contempla que, una vez cumplidos los anteriores, el conocimiento generado a través de la asociación entre la teoría estudiada en el aula y los datos empíricos obtenidos de esta salida a la realidad, se emplee como alternativa para gestionar cambios a nivel individual y social, referidos principalmente al cuidado de la salud y a la prevención de contingencias sanitarias, lo cual es una finalidad esencial presente en la salud pública, cuya historia se ha sintetizado en párrafos precedentes. En el transcurso de esa historia, se ha advertido cómo el motor de desarrollo de nuevos conocimientos en las disciplinas de la salud es justamente la resolución de problemas, por cuanto es un proceso que uti- 
liza el conocimiento de una disciplina para salvar el espacio existente entre el problema y su solución. Esta es una condición importante para el reconocimiento de la relevancia del conocimiento escolar, como señalan Zabala (2006). En el modelo de enseñanza que se propone, el alumno va de la definición del problema a su resolución, de forma que sea capaz de emitir hipótesis y diseñar estrategias o experiencias para su corroboración como el final del proceso.

Si como resultado de la indagación en el campo y la explicación con evidencia de los problemas de carácter individual y colectivos ejemplificados es posible aportar a la extensión del conocimiento en los estudiantes y a la toma de decisiones en los habitantes de una comunidad (por ejemplo, en el caso citado, exigir a su autoridad municipal que sea entubado el canal de aguas negras), el conocimiento adquiere una relevancia adicional, como medio ya no solo para comprender, sino para transformar el mundo. La implementación de un modelo de enseñanza como el esbozado en este trabajo puede generar en forma importante una transformación social, lo cual constituye una finalidad urgente en las ciencias biológicas y de la salud.

La educación que provoca el desarrollo de habilidades para pensar, para cuestionar, reside en la aplicación de estrategias de enseñanza basadas en la construcción del conocimiento, vinculadas con la realidad de su entorno. La teoría de la Modificabilidad Cognitiva constituye una herramienta trascendental desde su finalidad, al aplicar su Programa de Enriquecimiento Instrumental (PEI), que tiene como principio fundamental "Un momento, déjame pensar", el cuestionamiento y la mediación, así como el hacer consciente al educando de sus dificultades o deficiencias cognitivas para trabajar en ellas a manera de lograr plasticidad de su persona y facilitar e incrementar su aprendizaje (Feuerstein, 1994). La propuesta consiste en aplicar en la enseñanza de la biología o el resto de las áreas del conocimiento esta teoría pedagógica, resulta imprescindible que el docente se especialice en esta área pedagógica para enfrentar el reto, cambiar en sus estructuras cognitivas, animarse a provocar mediante el cuestionamiento y la mediación cambios en el aprendizaje, en la enseñanza de la biología y de la salud pública que buscan generar, a su vez, cambios de conductas de vida saludable, impactar positivamente en casos como el de la educación de la persona con diabetes u otros problemas de salud emergentes.

La teoría de Modificabilidad Estructural Cognitiva de Reuven-Feuerstein explica que el maestro es el principal agente de cambio y transformación de estructuras deficientes de alumnos con dificultades de aprendizaje; para ello, debe estar dotado de formación cognitiva, metodológica y ética humanística. El programa se basa en el principio de autoplasticidad cerebral y permite al sujeto adaptarse y ponerse al día con los cambios, preparándolo para enfrentar los retos del mundo globalizado. El instrumento de la modificabilidad cognitiva es el Programa de Enriquecimiento Instrumental, que consta de catorce instrumentos diseñados para modificar alguna función o estrategia cognitiva deficiente; busca modificar la autopercepción que el sujeto tiene y proporcionarle optimismo radical sobre sus posibilidades de cambio y mejora (Velarde, 2008).
De aquí la importancia de aplicar esta teoría en el proceso enseñanza-aprendizaje de la biología y de las ciencias de la salud pública en vísperas de encauzar la transformación del pensamiento y de la realidad socio-ambiental ante tantos problemas que acechan nuestro mundo globalizado. Esto significa que el conocimiento del contexto social resulta fundamental para el diseño de las estrategias que impacten en la salud pública, reconociendo la cultura, sus necesidades, sus enfermedades para cumplir el deber de ofrecer la posibilidad de servicios de salud (Ruvalcaba y Cortés Ascencio, 2012) y, evidentemente, brindar educación de calidad, que permita la construcción del conocimiento y con esto, a su vez, desarrollo social y mejora en su calidad de vida.

\section{Conclusiones}

La salud pública, por medio de sus acciones, ha impactado en cambios innegables. Basta con estudiar los datos respecto a niveles de mortalidad, la diversidad de padecimientos, el crecimiento de la población, el arsenal de la medicina o la complejidad de las instituciones de salud para cerciorarse de que la salud ha estado experimentando una vasta transición (Piédrola et al., 1991 y Frenk, 2003).

Lo anterior ha motivado la necesidad de personal cada vez más preparado y comprometido con la educación para la salud. El seguimiento de las condiciones del contexto socioambiental, en particular de las personas vulnerables, es otro de los cambios derivados principalmente del surgimiento de nuevas enfermedades causadas por virus y otros agentes con la capacidad de automodificarse de acuerdo con determinadas condiciones ambientales, casi siempre asociadas con la falta de formación de las personas para cuidar su medio y con la pobreza social, educativa y económica de las comunidades.

Se requiere, asimismo, de cambios en los métodos de enseñanza por parte de los educadores, propiciados en su génesis a partir del diseño de políticas públicas dirigidas a generar un alumno que piensa y construye en su clase, que, a su vez, permitan al educando desarrollar habilidades para pensar, el desarrollo de competencias aplicables en investigación, donde el cuestionamiento y la mediación constituyan el centro de la acción docente y además se vincule educación y salud desde el enfoque constructivista aplicado en biología, para que en un futuro la investigación biológica impacte con mejores efectos en la generación de una nueva salud pública.

La educación de calidad propicia el desarrollo poblacional, mismo que se requiere se inicie a partir de la aplicación de modelos pedagógicos desde el enfoque constructivista donde se construya el conocimiento y se estimule la colaboración, por ejemplo, el aprendizaje colaborativo grupal, el trabajo por proyectos, trabajo en equipo y la aplicaciones de teorías como la modificabilidad cognitiva a partir de la cual se propicie la generación de conciencia sobre su aprendizaje y sobre su entorno ambiental, para promover así la mejoría constante en estilos y calidad de vida. 


\section{Agradecimiento}

Los autores del presente artículo de investigación reconocemos y agradecemos profundamente la colaboración de la licenciada en inglés Yesenia Elizabeth Ruvalcaba Cobián por sus aportaciones en la revisión del artículo y traducción del abstract, situación que permite la posibilidad de incrementar la transferencia y modificación del conocimiento en el saber científico.

\section{Referencias bibliográficas}

Amory Winslow, E. (1923). The Evolution and Significance of the Modern Public Health Campaing. New Haven: Yale University Press.

Bogoya, D., Vinet, M., Restrepo, G. et al. (2000). Competencias y proyecto pedagógico. Bogotá: Universidad Pedagógica Nacional de Colombia.

Bonilla Pérez, G. A. y Vera Marín, V. (2011). ¿Cómo influye la educación ambiental en la cultura? Bio-grafía: Escritos sobre la biología y su enseñanza, 6(4), pp. 173-181.

Castillo Robles, M. G., Hernández Cota, V. y Robles González, T. (1998). Introducción a la Salud Pública. México: Instituto Politécnico Nacional.

Cortés Ascencio, S. y Ruvalcaba Ledezma, J. C. (2011a). La corresponsabilidad en salud pública. Viva Salud, pp. 40-43.

Cortés Ascencio S. y Ruvalcaba Ledezma, J. C. (2011b). Estrategias de educación para la salud pública. Viva Salud, , pp. 34-37.

Díaz Barriga, F. y Hernández, G. (2003). Paradigmas en psicología de la educación. México: Paidós.

Fajardo Ortiz, G., Carrillo, A. y Neri Vela, R. (2002). Perspectiva histórica de atención a la salud en México, 1902-2002. México: OPS y UNAM.

Felipe, A., Gallarreta, S. y Merino, G. (2005). La modelización en la enseñanza de la biología del desarrollo. Revista Electrónica de Enseñanza de las Ciencias, 3(4), pp. 1-33.

Feuerstein R. y Hoffman, M. B. (1994). Programa de enriquecimiento instrumental. Jerusalén: Editorial Bruño.

Freire, P. (1981). Pedagogía del oprimido. México: Siglo XXI.

Freire, P. (1983). La educación como práctica de la libertad. México: Siglo XXI.

Freire, P. (1991). Pedagogía de la autonomía. México: Siglo $\mathrm{XXI}$.

Frenk, J. (2003). La salud de la población, hacia una nueva salud pública. México: La ciencia para todos.

Frenk J., González-Pier, E., Gómez-Dantés, O., Lezana, M. y Knaul, F. (2007). Reforma integral para mejorar el desempeño del sistema de salud en México. Sal Pub Mex 2007, 49(1), pp. S23-S36.

González Panduro, A. (1999). Trascendencia y significado de la implementación del Programa de Enriquecimiento Instrumental en alumnos de nivel bachillerato. (Tesis de Licenciada en Educación Especial en el Área de problemas de Aprendizaje). Escuela Normal Superior de Especialidades.
Piédrola, G. et al. (1991). Medicina preventiva y salud pública. México: Ediciones Científicas y Técnicas.

Lazcano Ponce, E., Martínez Montañez, O. G., Charvel, S. y Hernández Ávila, M. (2013). Funciones esenciales de la salud pública, salud pública teoría y práctica. México: Editorial Manual Moderno.

López Luna, M. (1993). Salud pública. La evolución de la salud pública en México. México: Editorial Interamericana, McGraw-Hill.

Monereo, C. (2010). La formación del profesorado. Una pauta para el análisis e intervención a través de incidentes críticos. Revista Iberoamericana de Educación, 52, pp. 149-178.

Morin, Edgar (2007). La cabeza bien puesta. Repensar la reforma. Reformar el pensamiento, Madrid: Alianza.

Perrenoud, P. (2002). Construir competencias desde la escuela. Santiago de Chile: Dolmen.

Pozo, J. I. (1989). Teorías cognitivas del aprendizaje. Madrid: Morata.

Ruvalcaba Ledezma, J. C. (2002). Análisis de la práctica docente en la enseñanza de la ecología. Revista de la Universidad del Valle de Atemajac, 43(16), pp. 83-87.

Ruvalcaba Ledezma J.C. (2010). Entorno socio-ambiental, vital en la conservación de la salud. Aprehender. Consejo Municipal de Ciencia y Tecnología de Oaxaca de Juárez. Año II No. 7 agosto-septiembre, pp. 6-7.

Ruvalcaba Ledezma, J. C. y Cortés Ascencio, S. (2012). El sistema de salud mexicano. Odous Científica, 13(1), pp. 34-42. Recuperado de: http://servicio.bc.uc.edu.ve/odontologia/ revista/

Siguenza, A.F y Saenz., (1990). Análisis de la resolución de problemas como estrategia de enseñanza de la biología. Enseñanza de las Ciencias. 8 (3), 223-230.

Velarde Consoli, E. (2008). La teoría de la Modificabilidad Estructural Cognitiva de Reuven Feuerstein. Investigación Educativa, 22(12), pp. 203-221.

Zabala, A. (2006). Enfoque globalizadory pensamiento complejo. Barcelona: Graó. 